\title{
Optimising breast cancer screening reading: blinding the second reader to the first reader's decisions
}

\author{
Jennifer A. Cooper ${ }^{1,2} \cdot$ David Jenkinson $^{1} \cdot$ Chris Stinton ${ }^{1} \cdot$ Matthew G. Wallis ${ }^{3} \cdot$ Sue Hudson $^{4} \cdot$ Sian Taylor-Phillips $^{1,5}$ (D)
}

Received: 11 January 2021 / Revised: 2 March 2021 / Accepted: 30 March 2021 / Published online: 12 June 2021

(C) The Author(s) 2021

\begin{abstract}
Objectives In breast cancer screening, two readers separately examine each woman's mammograms for signs of cancer. We examined whether preventing the two readers from seeing each other's decisions (blinding) affects behaviour and outcomes.

Methods This cohort study used data from the CO-OPS breast-screening trial (1,119,191 women from 43 screening centres in England) where all discrepant readings were arbitrated. Multilevel models were fitted using Markov chain Monte Carlo to measure whether reader 2 conformed to the decisions of reader 1 when they were not blinded, and the effect of blinding on overall rates of recall for further tests and cancer detection. Differences in positive predictive value (PPV) were assessed using Pearson's chi-squared test. Results When reader 1 recalls, the probability of reader 2 also recalling was higher when not blinded than when blinded, suggesting readers may be influenced by the other's decision. Overall, women were less likely to be recalled when reader 2 was blinded (OR $0.923 ; 95 \%$ credible interval $0.864,0.986)$, with no clear pattern in cancer detection rate (OR 1.029; $95 \%$ credible interval 0.970, 1.089; Bayesian $p$ value 0.832 . PPV was $22.1 \%$ for blinded versus $20.6 \%$ for not blinded $(p<0.001)$. Conclusions Our results suggest that when not blinded, reader 2 is influenced by reader 1's decisions to recall (alliterative bias) which would result in bypassing arbitration and negate some of the benefits of double-reading. We found a relationship between blinding the second reader and slightly higher PPV of breast cancer screening, although this analysis may be confounded by other centre characteristics.

Key Points

- In Europe, it is recommended that breast screening mammograms are analysed by two readers but there is little evidence on the effect of 'blinding' the readers so they cannot see each other's decisions.

- We found evidence that when the second reader is not blinded, they are more likely to agree with a recall decision from the first reader and less likely to make an independent judgement (alliterative error). This may reduce overall accuracy through bypassing arbitration.

- This observational study suggests an association between blinding the second reader and higher positive predictive value of screening, but this may be confounded by centre characteristics.
\end{abstract}

Keywords Breast neoplasms $\cdot$ Mammography $\cdot$ Mass screening $\cdot$ Early detection of cancer $\cdot$ Markov chains

Sian Taylor-Phillips

s.taylor-phillips@warwick.ac.uk

1 Department of Health Sciences, Warwick Medical School, University of Warwick, Gibbet Hill Road, Coventry CV4 7AL, UK

2 Population Health Sciences; Bristol Medical School, University of Bristol, Bristol BS8 2BN, UK

3 Cambridge Breast Unit, Cambridge University Hospitals National Health Service Foundation Trust, and National Institute for Health Research Cambridge Biomedical Research Centre, Cambridge, UK

4 Peel \& Schriek Consulting Limited, London, UK

5 Warwick Screening, Warwick Medical School, University of Warwick, Coventry CV4 7AL, UK

\author{
Abbreviations \\ DCIS Ductal carcinoma in situ \\ DIC Deviance information criterion \\ ESS Effective sample size \\ MCMC Markov chain Monte Carlo \\ MLwiN Statistical Software Package for Fitting \\ Multilevel Models \\ NBSS National Breast Screening System \\ NPV Negative predictive value \\ PPV Positive predictive value \\ STROBE Strengthening the Reporting of Observational \\ Studies in Epidemiology
}




\section{Introduction}

Breast cancer screening is implemented in many European countries. European quality assurance guidelines recommend that mammograms are examined for signs of cancer by two radiologists (readers) using two mammographic views [1, 2]. There is evidence that this approach increases the cancer detection rate compared to single reading [3-7]. A retrospective analysis of women participating in the English NHS Breast Screening Programme identified that double reading with arbitration of discordant decisions reduced recall and increased cancer detection rates, compared to hypothetical single reading [7]. However, the cancers detected only by the second reader were smaller, had fewer involved nodes, and were of lower grade [7]. This finding is consistent with some prior research [8]. The identification of smaller lower grade cancers and DCIS may be beneficial, or it may not be a desirable outcome of breast cancer screening due to their association with overdiagnosis [9]. There is therefore currently debate about the efficacy of double reading [10].

An aspect of double reading that has received little research to date is the blinding of reader 2 to the decisions of reader 1 . Previous European guidance has recommended blinding, but the most recent version omits any recommendation on blinding except for in research studies [1,2]. There is some evidence that blinding may affect diagnostic accuracy and patient outcomes. One study investigated a consecutive series of mammograms from women participating in the national Dutch screening programme, with no arbitration of discordant results. This study found that blinded double reading resulted in higher programme sensitivity than non-blinded reading ( $83 \%$ vs $76 \%, p=0.01$ ) [11], albeit with higher benign biopsy rates when blinded (2.6 vs 1.4 per 1000 screens $p<0.001$ for ultrasound-guided core needle biopsy (CNB), and 5.9 vs 4.7 per 1000 screens $p=0.013$ for stereotactic CNB) [12]. These results suggest that reader 2 might be influenced by reader 1's decisions, but are not applicable to screening programmes which use arbitration of discordant reader decisions. The same study team produced some projections of the effect of blinding in this context, using a retrospective laboratory rather than clinical practice arbitration decision [13, 14].

In his monograph on errors in radiology, Smith [15] introduced the term 'alliterative error' to describe the influence that one radiologist can have on another. He suggested that, for example, if during an initial interpretation of a radiographic image an abnormality is missed, or a benign finding overemphasised, subsequent interpretations may lead to the same erroneous conclusion. This can occur when the subsequent interpretation of the original image is carried out by a different reader or the original reader. Smith proposed that this may occur because the second reader reads the results of previous examinations before making their own decision, and then tend to adopt the same position, conforming to the belief of their peers. While there have been few published studies of alliterative errors, it is often reported as a source of error in radiology [16-19].

If non-blinded decision-making can introduce alliterative bias, this could affect rates of recall, cancer detection, and outcomes for women attending screening. Optimising reading conditions could improve the balance of benefits and harms of breast screening. The aim of this research was to determine the effect of blinding the second reader in breast cancer screening on alliterative error and subsequently the effect on screening accuracy (recall and cancer detection rate), in a population breast screening programme which uses arbitration of discrepant reader decisions.

\section{Materials and methods}

\section{Study design}

This study is reported using the 'STROBE' statement [20]. The study is a population-based cohort study within the Changing Case Order to Optimise Patterns of Performance in Screening (CO-OPS) trial. The original trial investigated patterns of performance and fatigue with time on task, and is described in detail elsewhere [21]. Briefly, the trial included 1,194,147 women (predominantly aged 47-73 years) attending routine triennial digital mammography screening between December 2012 and November 2014 at 46 English centres. Women with high-familial risk and who presented symptomatically were excluded. Digital mammograms were assessed independently by two expert readers (radiologists, radiography advanced practitioners, breast clinicians) for signs of cancer and whether a woman should be recalled for further investigation. Readers in the screening programme are required to examine a minimum of 5000 mammograms a year and have undergone extensive training [22]. Arbitration was used at all centres when there were disagreements between the two readers ( 13 centres used a third reader, 33 used group consensus of 2 or more readers). Additionally, at some centres, arbitration was used even when both readers suggested recall, in an effort to reduce overall recall rates. The National Breast Screening Service (NBSS) database records the decisions of the readers and clinical information for each woman.

\section{Data collection}

Data were extracted from the NBSS system. Fields which indicate the 'blind' status at the time reader 1 and reader 2 saved their opinions were extracted. Reader 1 selects whether reading is blinded and then the second reader can change this during their reading session. When the blind reporting option is selected in NBSS, it masks the opinions of the previous reader by showing 'Entered' in place of the opinions. In the blinded reading condition, reader 2 could still ascertain what 
reader 1 decided by looking through the paper notes; however, this would be rare due to time constraints in the high-volume screening environment.

\section{Statistical analysis}

Summary statistics of the characteristics of the women screened and the outcomes by the first reader, second reader and after arbitration of discordant decisions were presented by whether reader 2 was blinded. To investigate whether alliterative bias was present, we compared the proportion of cases where there were discordant decisions between readers using a chi-squared test. The hypothesis was that blinding the second reader would increase disagreements by reducing alliterative bias. We then directly modelled whether the second reader was influenced by the first reader's decision when not blinded, i.e. whether alliterative bias was present. The model outcome was the second reader decision, with fixed effects for whether reader 1 recalled the woman and whether reader 2 was blinded, and an interaction term between them.

We fitted a multi-level model using Markov chain Monte Carlo (MCMC) methods using R2MLwiN [23], which runs the multilevel modelling program MLwiN [24, 25] from within the ' $\mathrm{R}$ ' environment. A MCMC approach provides several advantages over maximum likelihood estimation in this context. It can achieve more accurate model estimates particularly with more complex models and gives a posterior probability distribution for the parameters, rather than a $p$ value [26-28]. The unit of analysis was the woman screened, with clustering by reader and centre. We included fixed effects for whether a woman was attending her first or a subsequent screen and the woman's age (continuous, centred). Random effects were included for the second reader (level 2) and screening centre (level 3).

To investigate whether any alliterative bias may affect screening accuracy, we modelled whether blinding the second reader was associated with differences in overall recall and cancer detection rates. Two interaction terms were considered for inclusion, based on the Bayesian deviance information criterion (DIC) to assess overall model fit and the $p$ value of the z-score for an estimate (5\% level) [23]. An interaction between blinding and age was included because younger women tend to have a higher density of breast tissue, increasing task difficulty [29]. An interaction between blinding and previous screening attendance was assessed because a lack of previous mammograms for comparison also increases task difficulty. Cancer detection and recall rate for reader 2 (without arbitration) were also modelled to assess the intervention effect (Supplementary Material Appendix A).

Tumour characteristics (DCIS grade, disease grade, invasive disease presence, number of positive axillary nodes, maximum diameter of invasive disease) were determined for blinded/non-blinded reader 2 with statistical testing $\left(\chi^{2}\right.$ test for independence, test for equality of two proportions and $t$ test) to determine any significant differences. The positive predictive value (PPV) when blinding the second reader compared to not blinding was reported, using the reference standard of biopsy-proven cancer after recall from screening. Pearson's chi-squared test was used to compare PPV in cases read blinded and not blinded. To assess the potential impact of centre confounding (fully blinded centre, vs fully non-blinded, vs mixed centres), all the above models were run with a subset of six centres which had a mix of blinded and non-blinded reading as a sensitivity analysis. A mixed protocol centre was one where there was at least $5 \%$ of blinded or not blinded out of the total number of mammograms read at the centre (Supplementary Material Appendix C).

Interval cancers within 3 years of screening were used to estimate test accuracy metrics for blinded/non-blinded reading (sensitivity, specificity, PPV, negative predictive value (NPV)). We separated the women not recalled into 'false negatives' (women not recalled who had an interval cancer within 3 years of screening) and 'true negatives' (women not recalled and either did not have an interval cancer recorded in their follow-up data or did not have follow-up data). For consistency within this analysis, anyone recalled, had no cancer detected, and had an interval cancer within 3 years of screen was classified as a true positive, rather than a false positive. We performed an equality of proportions test to determine whether these were statistically significant (Supplementary Material Appendix E).

\section{Results}

\section{Descriptive statistics}

A total of 1,119,191 women were included from 43 screening centres with 9656 cancers detected after arbitration $(0.86 \%)$. The mean age of the women was 59 , and $78.8 \%$ had previously attended screening $(881,900 / 1,119,191)$. The study flow diagram is depicted in Fig. 1. Study characteristics and outcomes by blinding status are presented in Table 1 . Of the 43 centres, 23 centres were classified as not blinded, 14 as blinded, and 6 as mixed. There were 418 first readers and 420 second readers. Reader 2 was blinded for $34.2 \%$ of women screened.

\section{Alliterative bias}

Rates of disagreement between the two readers for recall were $0.20 \%$ points higher when blinded $(3.57 \%$; $95 \%$ CI: $3.51 \%$, $3.63 \%)$ than when not $(3.37 \%$; $95 \%$ CI: $3.33 \%, 3.41 \%)\left(\chi^{2}(1)\right.$ $=32.46, p<0.001)$. The disagreement rate difference increases to $5.60 \%$ points when the first reader recalls the case (38.61\%; 95\% CI: $37.92 \%, 39.29 \%$ ) when reader 2 is blinded 
Fig. 1 Study flow diagram with reasons for exclusions. There were 46 centres in the CO-OPS trial, but three shared a common computer system so are counted as one centre in this analysis, a further centre was removed which had no reader identifiers, giving 43 centres in the dataset. Of the 43 centres, 23 centres were classified as not blinded, 14 as blinded, and 6 as mixed. Reader 2 was blinded for $34.2 \%(382,490 / 1,119,191)$ of women screened

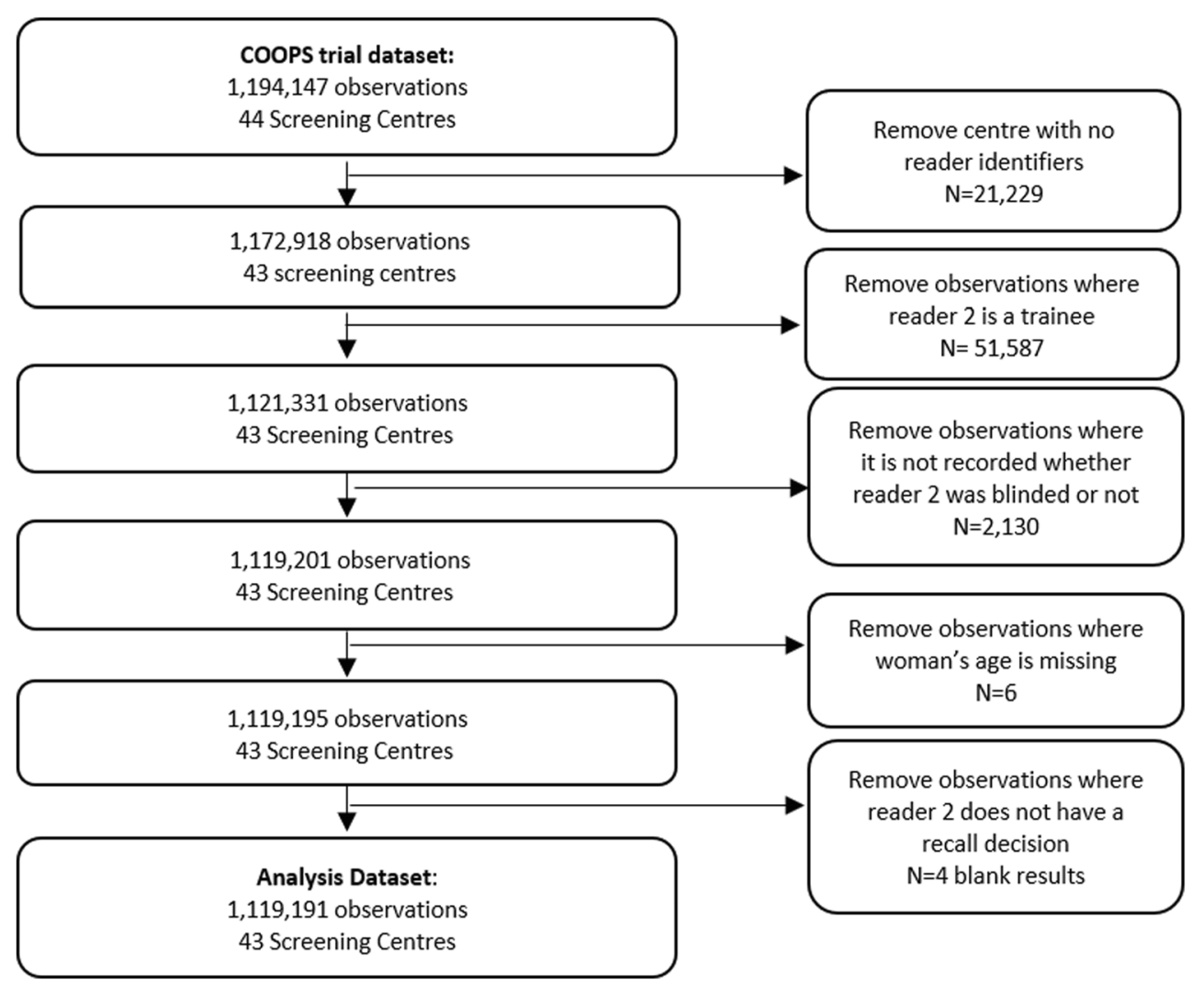

versus $(33.01 \%$; $95 \% \mathrm{CI}: 32.55 \%, 33.48 \%)$ when reader 2 is not blinded $\left(\chi^{2}(1)=179.03, p<0.001\right)$ (Supplementary Table B.2).

The multilevel model results show a similar pattern (Fig. 2). When reader 1 recalls, the probability of reader 2 recalling is $4.9 \%$ points lower when blinded $(69.8 \%$; $95 \%$ credible interval: $67.9 \%, 71.5 \%)$ versus not blinded $(74.7 \%$; $95 \%$ credible interval: $73.2 \%, 76.1 \%$ ) for a woman's first screen. If reader 1 does not recall, the probability of reader 2 recalling when blinded (2.33\%; $95 \%$ credible interval: $2.14,2.53 \%$ ) and not blinded (2.32\%; $95 \%$ credible interval: $2.15,2.50 \%)$ is similar for a woman's first screen. The model and full probabilities are reported in Supplementary Tables B.1 and B.3.

\section{Recall rate, cancer detection rate, and PPV}

\section{Recall rate}

Reader 2 (before arbitration) recalled $0.45 \%$ points fewer women when blinded $(4.73 \%$; $95 \%$ CI: $4.67 \%, 4.80 \%)$ than when not blinded $(5.18 \%$; $95 \%$ CI: $5.13 \%, 5.23 \%)\left(\chi^{2}(1)=\right.$ $107.04, p<0.001$ ). However, reader 1 (who cannot see reader 2 's decision, as by definition it does not yet exist) also recalled fewer women $(0.26 \%$ points $)$ when reader 2 was blinded $(5.09 \%$; $95 \%$ CI: $5.02 \%, 5.16 \%)$ than when not blinded $(5.35 \%$; $95 \%$ CI: $5.30 \%, 5.40 \%)\left(\chi^{2}(1)=34.751, p<0.001\right)$ indicating at least part of this effect may be due to confounding (Table 1).

Recall rate after arbitration was lower when reader 2 was blinded (3.96\%; 95\% CI: $3.90 \%, 4.02 \%)$ compared to when they were not $(4.16 \%$; $95 \%$ CI: $3.93 \%, 4.38 \%)\left(\chi^{2}(1)=24.51\right.$, $p<0.001)$. A model assessing the effect of blinding reader 2 on the recall rate after arbitration is presented in Table 2 and Fig. 3. Blinding reader 2 decreased the odds of recall from screening compared to not blinding (OR 0.869; $95 \%$ credible interval $0.826,0.913$ ) for a woman of average age (59 years) who has previously been screened (Table 2).

The interactions for the recall rate model were dissected in an interaction plot (Fig. 3). Blinding reader 2 decreased the odds of recall after arbitration for both first time and subsequent screens, and for all ages. The trend was towards a greater effect of blinding on recall rate at younger ages, and when the woman had previously attended screening. For both first and subsequent screen mammograms of 60 -year-old women, women were less likely to be recalled if reader 2 was blinded than if they were not: first screen OR 0.923 (95\% credible interval $0.864,0.986$ ), subsequent screen OR 0.871 (95\% credible interval $0.829,0.915$ ) (Fig. 2, Table A.2 Supplementary Material).

Analysis of the subset of 179,573 women at the six centres in which there was a mixture of blinded/unblinded second readers showed similar results. Blinding the second reader 
Table 1 Characteristics of the study sample, and recall and cancer detection rates for blinded versus not blinded reader 2

\begin{tabular}{|c|c|c|c|c|c|}
\hline \multicolumn{2}{|l|}{ Study characteristic } & \multirow{2}{*}{$\begin{array}{l}\begin{array}{l}\text { Reader } 2 \\
\text { Blinded }\end{array} \\
59.2\end{array}$} & \multirow[t]{2}{*}{$\%$} & \multirow{2}{*}{$\begin{array}{l}\begin{array}{l}\text { Reader } 2 \\
\text { Not blinded }\end{array} \\
59.3\end{array}$} & \multirow[t]{2}{*}{$\%$} \\
\hline Age of woman (mean) & Mean age & & & & \\
\hline & Group 1 (52 or less) & 90,505 & 23.66 & 167,217 & 22.70 \\
\hline & Group $2(53-59)$ & 111,642 & 29.19 & 214,996 & 29.18 \\
\hline & Group 3 (60 or more) & 180,343 & 47.15 & 354,488 & 48.12 \\
\hline & Total & 382,490 & 100.00 & 736,701 & 100.00 \\
\hline \multirow[t]{3}{*}{ First screen/subsequent screen } & Subsequent screen & 300,820 & 78.65 & 581,080 & 78.88 \\
\hline & First screen & 81,670 & 21.35 & 155,621 & 21.12 \\
\hline & Total & 382,490 & 100.00 & 736,701 & 100.00 \\
\hline Reader 1 & Not recalled & 363,034 & 94.91 & 697,294 & 94.65 \\
\hline \multirow[t]{2}{*}{ Recall (pre arbitration) } & Recalled & 19,456 & 5.09 & 39,407 & 5.35 \\
\hline & Total & 382,490 & 100.00 & 736,701 & 100.00 \\
\hline Reader 2 & Not recalled & 364,387 & 95.27 & 698,512 & 94.82 \\
\hline \multirow[t]{2}{*}{ Recall (pre arbitration) } & Recalled & 18,103 & 4.73 & 38,189 & 5.18 \\
\hline & Total & 382,490 & 100.00 & 736,701 & 100.00 \\
\hline \multirow[t]{3}{*}{ Recall (after arbitration) } & Not recalled & 367,341 & 96.04 & 706,082 & 95.84 \\
\hline & Recalled & 15,149 & 3.96 & 30,619 & 4.16 \\
\hline & Total & 382,490 & 100.00 & 736,701 & 100.00 \\
\hline \multirow[t]{3}{*}{ Cancers detected by reader 1} & Cancer detected & 3066 & 0.80 & 5717 & 0.78 \\
\hline & No Cancer detected & 379,424 & 99.20 & 730,984 & 99.22 \\
\hline & Total & 382,490 & 100.00 & 736,701 & 100.00 \\
\hline \multirow[t]{3}{*}{ Cancers detected by reader 2} & Cancer detected & 3226 & 0.84 & 6117 & 0.83 \\
\hline & No cancer detected & 379,264 & 99.16 & 730,584 & 99.17 \\
\hline & Total & 382,490 & 100.00 & 736,701 & 100.00 \\
\hline \multirow{3}{*}{$\begin{array}{l}\text { Cancers detected overall } \\
\text { (after arbitration) }\end{array}$} & Cancer detected & 3355 & 0.88 & 6301 & 0.86 \\
\hline & No Cancer detected & 379,135 & 99.12 & 730,400 & 99.14 \\
\hline & Total & 382,490 & 100.00 & 736,701 & 100.00 \\
\hline
\end{tabular}

was associated with a lower recall rate after arbitration than when the second reader was not blinded (OR 0.883; 95\% credible interval 0.834, 0.933) (Supplementary Table C.2).

\section{Cancer detection rate}

Reader 2 (before arbitration) detected cancers in $0.84 \%(95 \%$ CI: $0.81 \%, 0.87 \%$ ) of women when blinded versus $0.83 \%$ (95\% CI: $0.81 \%, 0.85 \%)$ when not blinded $\left(\chi^{2}(1)=0.51\right.$, $p=0.48)$. The cancer detection rate overall (with arbitration) was similar when reader 2 was blinded $(0.88 \%$; $95 \% \mathrm{CI}$ : $0.85 \%, 0.91 \%)$ compared to when not $(0.86 \% ; 95 \% \mathrm{CI}$ : $\left.0.83 \%, 0.88 \%, \chi^{2}(1)=1.4, p=0.2\right)$.

The model determining the association of blinding with cancer detection rate after arbitration is reported in Table 3. The association between blinding and cancer detection was not statistically significant (OR 1.029; $95 \%$ credible interval: $0.970,1.089 ; p=0.341)$, although the Bayesian $p$ value suggests that $83.2 \%$ of estimates lie above an odds ratio of 1 (showing a potential positive association) (Supplementary Material Table A.5). Cancer detection also increases with age and with a first screen versus a subsequent screen.

Analysis of the subset of six centres (179,573 women) where there is a mix of blinded/unblinded second readers showed similar results (Supplementary Table C.4).

\section{Tumour characteristics}

Tumour characteristics by whether reader 2 is blinded or not is shown in Supplementary Table D.1. Invasive disease was present in $78.4 \%(2570 / 3277)$ for blinded and $76.6 \%$ (4503/5881) for not blinded $\left(\chi^{2}(1)=4.0, p=0.0449\right)$; with no significant evidence of any difference for disease grade $\left(\chi^{2}(2)=0.67, p=0.7\right)$, the number of positive axillary nodes $\left(\chi^{2}(2)=3.0, p=0.2\right)$, and the mean diameter of the tumour for blinded $(16.5 \mathrm{~mm}$, standard deviation (SD) 12.6 $\mathrm{mm}$ ) and not blinded (16.2 mm, SD $11.8 \mathrm{~mm} ; \mathrm{t}=1.0, p=$ $0.3)$. When invasive disease was not present, there was no evidence of an effect of blinding on grade of DCIS $\left(\chi^{2}(2)=1.99, p=0.37\right)$. 

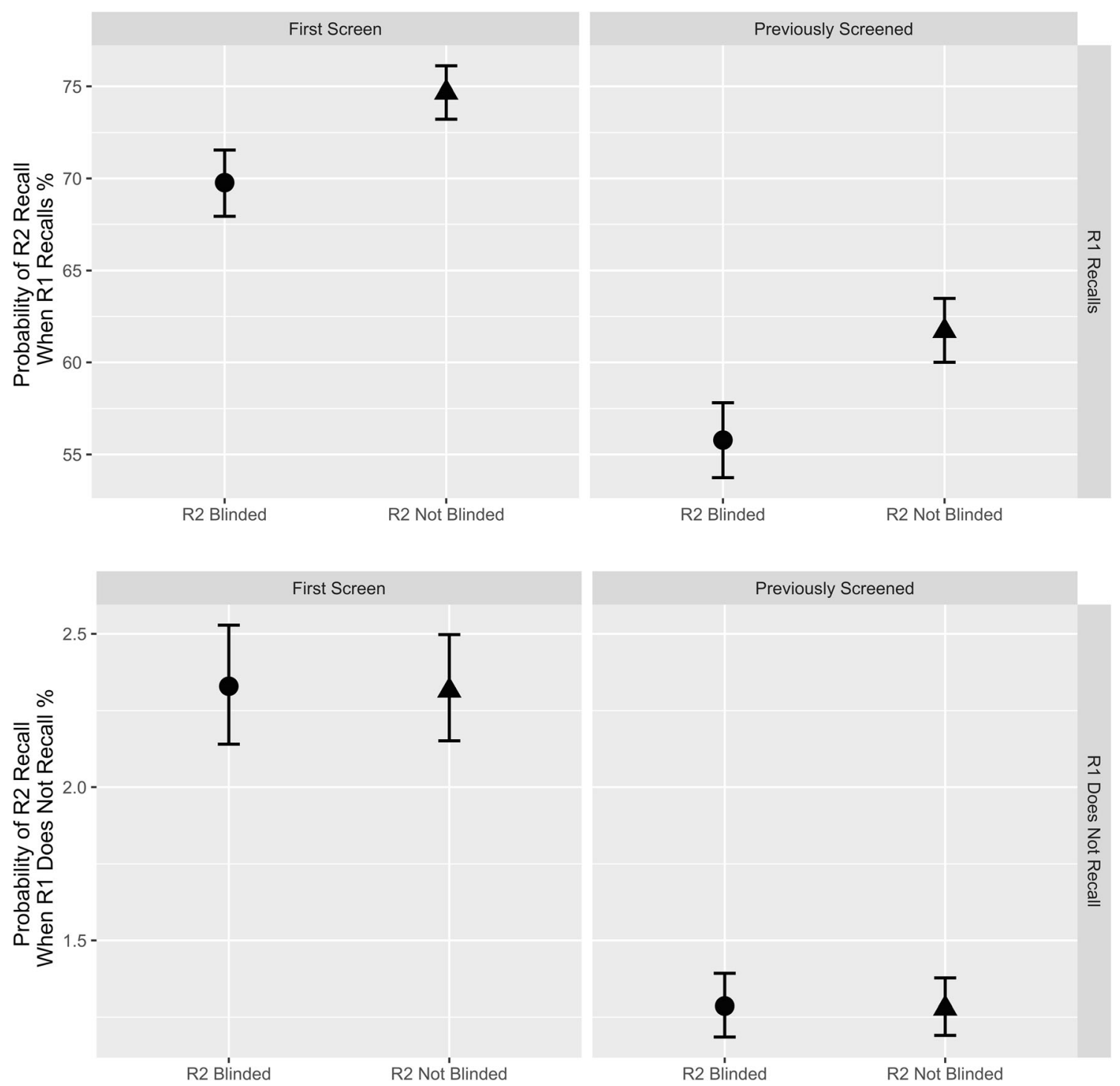

Fig. 2 Facet plot showing the probability (with $95 \%$ credible interval) of reader 2 recalling a woman (at the mean age of 59.27) by whether reader 1 (R1) recalls or not, when reader 2 (R2) is blinded and not blinded. Results

also presented for women who are first time screens or subsequent screens. The probability of recall is lower for a woman attending a subsequent screen compared to attending a first-time screen
Table 2 Markov chain Monte Carlo (MCMC) multilevel model determining the effect of blinding on recall rate overall (after arbitration). The full model including one-sided Bayesian $p$ values is reported in the Supplementary Material (Table A.1) along with the caterpillar plot showing level 3 residuals and their $95 \%$ CIs (Figure A.1)
Recall rate overall (after arbitration) multilevel model

\begin{tabular}{|c|c|c|c|}
\hline Fixed Effects & Odds ratio $^{a}$ & $\begin{array}{l}95 \% \text { credible interval } \\
\text { for the odds ratio }{ }^{\mathrm{b}}\end{array}$ & $\operatorname{Pr}(>|z|)^{\mathbf{c}}$ \\
\hline Blinding Yes (versus no as the reference category) & 0.869 & $0.826,0.913$ & $<0.001$ \\
\hline Age (centred) & 1.007 & $1.005,1.009$ & $<0.001$ \\
\hline $\begin{array}{l}\text { First screen (versus subsequent screen as the } \\
\text { reference category) }\end{array}$ & 2.823 & $2.728,2.922$ & $<0.001$ \\
\hline Blinded yes $*$ age (interaction term) & 1.005 & $1.001,1.008$ & 0.015 \\
\hline Blinding yes $*$ first screen (interaction term) & 1.060 & $0.999,1.125$ & 0.057 \\
\hline
\end{tabular}

a The mean of the 100,000 chain iterations after converting from the log odds scale to the odds scale.

b $95 \%$ credible interval is generated by taking the 2.5 th and 97.5 th quantiles of the 100,000 chain iterations after converting from the log odds scale to the odds scale

${ }^{\mathbf{c}}$ Two-tailed $p$ value of the $\mathrm{z}$ score for the coefficient (testing whether the estimate is significantly different from zero assuming normality) 
Fig. 3 Interaction plot displaying the odds ratios (median and $95 \%$ credible intervals) of recall rate after arbitration when reader 2 is blinded versus not blinded by screen status and age. See accompanying Table A.2 in the Supplementary Material

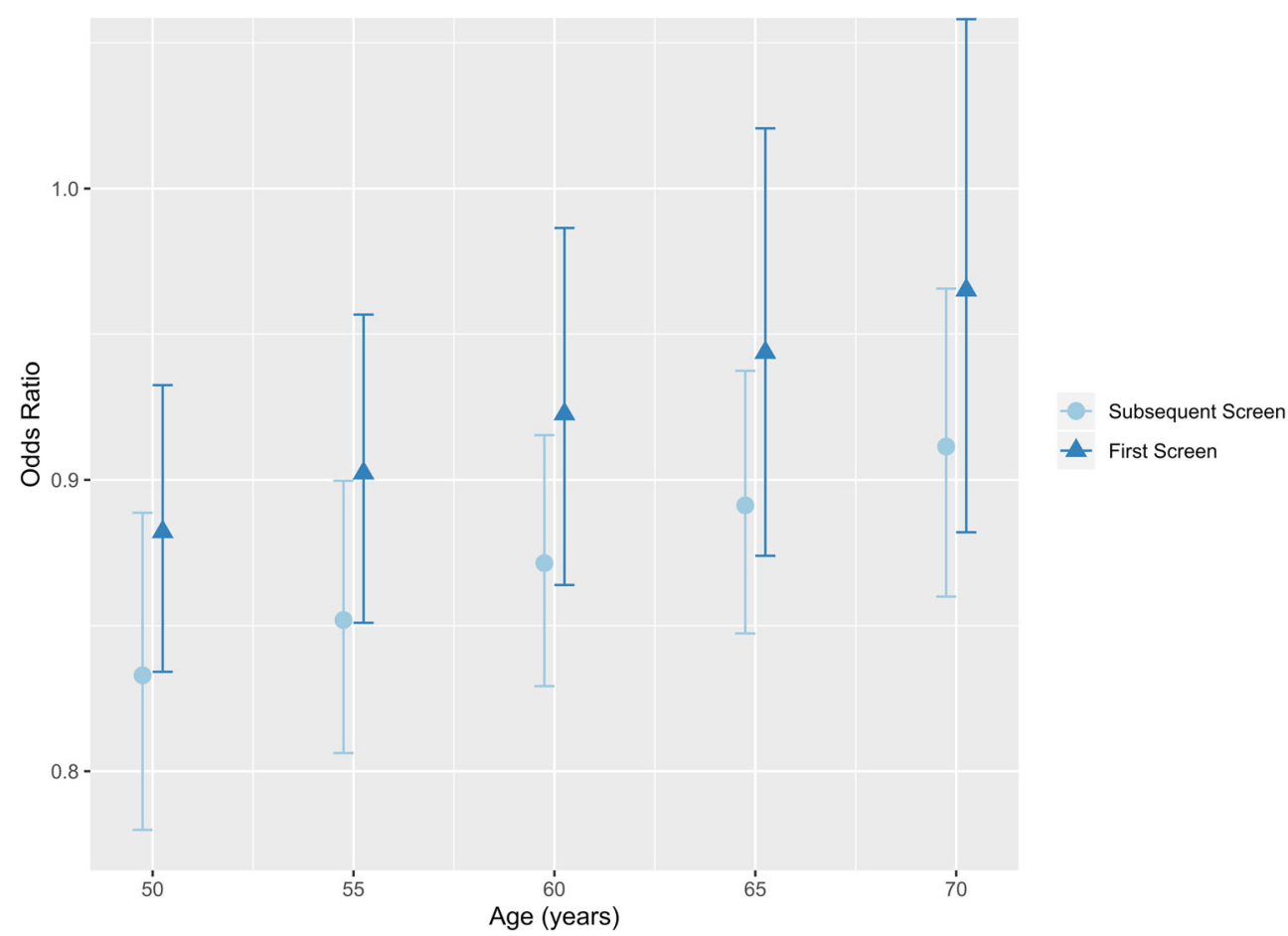

\section{Positive predictive value}

The PPV after arbitration was $22.1 \%(3355 / 15,149 ; 95 \%$ CI: $21.5 \%, 22.8 \%)$ for blinded and $20.6 \%(6301 / 30,619 ; 95 \% \mathrm{CI}$ : $20.1 \%, 21.0 \%)$ when not blinded $\left(\chi^{2}(1)=14.9, p<0.001\right)$ (Table 4).

\section{Further test accuracy estimates based on interval cancer data}

Interval cancers within 3 years of screening were used to estimate test accuracy metrics and are shown in Table 5. The estimated sensitivity was $82.44 \%$ (3399/4123; 95\% CI: $81.28 \%, 83.60 \%)$ for blinded and $82.60 \%$ (6391/7737; $95 \%$

Table 3 Markov chain Monte Carlo (MCMC) multilevel model determining the effect of blinding on cancers detected overall (after arbitration). Interactions were not included for this model due to both a higher deviance information criterion (DIC) in comparison to a model without
CI: $81.76 \%, 83.45 \%)$ for not blinded $\left(\chi^{2}(1)=0.04, p=\right.$ $0.84)$. The difference for specificity was statistically significant: $96.89 \%$ for blinded and $96.68 \%$ for not blinded $\left(\chi^{2}(1)=37.6, p<0.001\right)$.

\section{Discussion}

\section{Summary of results}

We examined the effect that blinding reader 2 to the decision of reader 1 had on behaviour and outcomes using data from the English Breast Cancer Screening Programme. When reader 1

and non-significant $p$ values for the estimates. The full model including one-sided Bayesian $p$ values is reported in the Supplementary Material (Table A.5) along with the caterpillar plot showing level 3 residuals and their 95\% CIs (Figure A.3)

Cancer detection overall (after arbitration) multilevel model

\begin{tabular}{lllr}
\hline Fixed effects & Odds ratio $^{\mathrm{a}}$ & $95 \%$ credible interval for the odds ratio $^{\mathrm{b}}$ & $\operatorname{Pr}^{\mathrm{b}(>|\mathrm{z}|)^{\mathrm{c}}}$ \\
\hline Blinding yes (versus no as the reference category) & 1.029 & $0.970,1.089$ & 0.341 \\
Age (centred) & 1.052 & $1.049,1.056$ & $<0.001$ \\
First screen (versus subsequent screen as the reference category) & 1.696 & $1.591,1.807$ & $<0.001$
\end{tabular}

${ }^{\mathbf{a}}$ The mean of the 100,000 chain iterations after converting from the log odds scale to the odds scale

b $95 \%$ credible interval is generated by taking the 2.5 th and 97.5 th quantiles of the 100,000 chain iterations after converting from the log odds scale to the odds scale

${ }^{\mathbf{c}}$ Two-tailed $p$ value of the $\mathrm{z}$ score for the coefficient (testing whether the estimate is significantly different from 0 assuming normality) 
Table 4 Two by two table of positive predictive value (PPV) for both after arbitration and for reader 2 against whether reader 2 is blinded or not. Chi-squared test for independence assesses whether PPV after arbitration is independent of blinding $\left(\chi^{2}(1)=14.9\right.$ $p<0.001)$ and whether PPV of reader 2 is independent of blinding $\left(\chi^{2}(1)=28.7, p<0.001\right)$

\begin{tabular}{llll}
\hline & $\begin{array}{l}\text { Reader 2 } \\
\text { Blinded }\end{array}$ & $\begin{array}{l}\text { Reader 2 } \\
\text { Not blinded }\end{array}$ & Total \\
\hline Cancer detected (TP) after arbitration & 3355 & 6301 & 9656 \\
Recall (after arbitration), no cancer detected (FP) & 11,794 & 24,318 & 36,112 \\
Total recalled (TP + FP) & 15,149 & 30,619 & 45,768 \\
PPV (TP/total recalled) \% & $22.1 \%$ & $20.6 \%$ & - \\
& & & 9343 \\
Cancer detected (TP) (reader 2) & 3226 & 6117 & 46,949 \\
Recall (reader 2), no cancer detected (FP) & 14,877 & 32,072 & 56,292 \\
Total recalled (TP + FP) & 18,103 & 38,189 & - \\
PPV (TP/total recalled)\% & $17.8 \%$ & $16.0 \%$ & \\
\hline
\end{tabular}

recalled, the probability of reader 2 recalling was around 5\% points lower when blinded versus not (69.8\% vs $74.7 \%)$, suggesting that without blinding they are influenced by the decision of reader 1 and alliterative bias is present. This has the potential to increase recall rates by bypassing arbitration in systems where there is arbitration of discordant decisions. We found that the overall odds of recalling women for further tests were lower and specificity was higher when reader 2 was blinded to the decision of reader 1 compared to when not blinded. Similarly, the PPV after arbitration when reader 2 was blinded was slightly higher $(22.1 \%)$ versus when not $(20.6 \%, p<0.001)$. We also found a difference (albeit smaller) in reader 1 recall rates when reader 2 was blinded versus unblinded. This may be due to reader 1 changing their behaviour in anticipation of reader 2 viewing their decision, a training effect from independent reading, or it may be an indication of centre level confounding.

\section{Comparison with the literature}

We identified only one study that directly statistically compared the effects of blinding reader 2 compared to not blinding reader 2 in the setting of a breast cancer screening programme [11]. This study used a system of recalling all discordant results. Klompenhouwer et al [11] found that when reader 2 was not informed of the decision of reader 1 , the sensitivity of the screening programme was higher $(83.1 \%$ vs $75.5 \%)$, recall rate was higher $(3.3 \%$ vs $2.9 \%)$, false positive referrals were higher (2.6\% vs $2.2 \%$ ), and the interval cancer rate was lower (1.5 per 1000 screens vs 2.1 per 1000 screens). There was no difference in PPV, cancer detection rate, or proportion of BIRADS 4 or 5 . This provides some evidence of the impact of blinding, but is not applicable to screening programmes where discordant decisions are arbitrated.

Table 5 Test accuracy statistic estimates for reader 2 blinded or not blinded. Cancer includes those detected at the screen and interval cancers within 3 years of the screen

\begin{tabular}{|c|c|c|c|c|c|c|c|c|c|c|c|}
\hline & & \multirow{2}{*}{\multicolumn{4}{|c|}{$\begin{array}{l}\text { Reader } 2 \\
\text { Blinded } \\
\text { Cancer }\end{array}$}} & \multicolumn{4}{|c|}{$\begin{array}{l}\text { Reader } 2 \\
\text { Not blinded }\end{array}$} & \\
\hline & & & & & & \multicolumn{4}{|l|}{ Cancer } & \multicolumn{2}{|c|}{$\begin{array}{l}\text { Equality of } \\
\text { proportions tests }\end{array}$} \\
\hline & & Detected & Interval & None & Total & Detected & Interval & None & Total & & \\
\hline \multirow[t]{3}{*}{ Recall (after arbitration) } & Yes & 3355 & 44 & 11,750 & 15,149 & 6301 & 90 & 24,228 & 30,619 & - & \\
\hline & No & 0 & 724 & 366,617 & 367,341 & 0 & 1346 & 704,736 & 706,082 & - & \\
\hline & Total & 3355 & 768 & 378,367 & 382,490 & 6301 & 1436 & 728,964 & 736,701 & - & \\
\hline \multicolumn{4}{|l|}{ Test accuracy statistics } & \multicolumn{2}{|c|}{$95 \%$ confidence interval } & & & \multicolumn{2}{|c|}{$95 \%$ confidence interval } & $\chi^{2}(1)$ & $p$ \\
\hline Sensitivity & & \multicolumn{2}{|l|}{$82.44 \%$} & \multicolumn{2}{|c|}{$(81.28 \%, 83.60 \%)$} & \multicolumn{2}{|l|}{$82.60 \%$} & \multicolumn{2}{|c|}{$(81.76 \%, 83.45 \%)$} & 0.0390 & 0.843 \\
\hline Specificity & & \multicolumn{2}{|l|}{$96.89 \%$} & \multicolumn{2}{|c|}{$(96.84 \%, 96.95 \%)$} & \multicolumn{2}{|l|}{$96.68 \%$} & \multicolumn{2}{|c|}{$(96.64 \%, 96.72 \%)$} & 37.6 & $<0.001$ \\
\hline PPV & & \multicolumn{2}{|l|}{$22.44 \%$} & \multicolumn{2}{|c|}{$(21.77 \%, 23.10 \%)$} & \multicolumn{2}{|l|}{$20.87 \%$} & \multicolumn{2}{|c|}{$(20.42 \%, 21.33 \%)$} & 14.7 & $<0.001$ \\
\hline NPV & & \multicolumn{2}{|l|}{$99.80 \%$} & \multicolumn{2}{|c|}{$(99.79 \%, 99.82 \%)$} & \multicolumn{2}{|l|}{$99.81 \%$} & \multicolumn{2}{|c|}{$(99.80 \%, 99.82 \%)$} & 0.491 & 0.483 \\
\hline
\end{tabular}

$P P V$ positive predictive value, $N P V$ negative predictive value

Number of women with cancer used in the "test accuracy" statistics found by adding the detected and interval cancers together 
Follow on studies assessed the impact of arbitration versus no arbitration of discrepant readings for both blinded and non-blinded reading $[13,14]$. To do this, they randomly assigned a third reader to decide retrospectively whether to recall a discrepant reading [13]. Although blinded double reading with arbitration was not directly statistically compared to non-blinded double reading with arbitration, the recall rate was lower for blinded reading $2.2 \%$ versus $2.3 \%$ for non-blinded reading, PPV was higher $31.2 \%$ compared to $27.5 \%$, and cancer detection rate was 6.8 per 1000 screens versus 6.3 per 1000 screens with the proportion of BI-RADS 0 (low suspicion lesions) among recalls at $23.0 \%$ versus $26.7 \%$. Sensitivity was $76 \%$ for blinded versus $72.7 \%$. Our results show this effect of increased PPV and decreased recall rate with blinding is present also in clinical practice, and is statistically significant. Both studies are inconclusive on the effect of blinding on cancer detection and sensitivity, with trends towards increases which are not statistically significant.

In summary, the previous studies in the Dutch screening programme have shown that when all discordant decisions are recalled, blinding increases cancers detected at screening, and number of false positive recalls to assessment, but with similar PPV. They projected that in screening programmes with arbitration blinding may increase PPV; this was a retrospective analysis rather than prospective measurement. Our study findings aligns with these and expands them. In clinical practice where arbitration is used, our study suggests that blinding improves PPV through increases to specificity. We also found evidence of alliterative bias, which explains the mechanism of action of these effects.

\section{Strengths and limitations}

This study has a number of key strengths. For example, we used a large dataset that was collected as part of a breast screening programme, which included a representative sample of screening centres and women in England, and had very little missing data. We also used a Bayesian approach to modelling, fitting models with MCMC methods. These methods generate a sample from the posterior probability distribution of the parameter which can then be summarised by giving the probability of the coefficient being greater/smaller than 0 . This enabled us to assess whether the evidence was compelling enough that the cancer detection rate may increase when the second reader is blinded. Overreliance on the use of a statistically significant cut-off level under frequentist inference may lead to the dismissal of clinically relevant effects [26-28]. Our research provided Bayesian $p$ values as an additional measure which can convey the strength of the blinding effect.

The study has limitations. Our data are observational, so we cannot conclude that blinding is causing the improvement in PPV and reduction in recall. Reader 2 was also shown to perform better than reader 1 under both blinded and non-blinded conditions, suggesting that potentially more experienced and senior readers read second more frequently. To reduce this potential bias, trainee readers were removed from the population sample. In this study, we measured readers' decisions and the woman's outcomes, but no measurements were made of reading behaviour or how the second reader may have used the first reader's decision. The blinded versus non-blinded improvement is also seen to a lesser extent in reader 1 which cannot be caused by the alliterative effect. Services that used blinding could have more experienced readers overall or could serve a different population demographic of screened women (e.g. by ethnicity, socioeconomic status). Differences between centres were however addressed by clustering by centre and reader as well as controlling by age and screening status. Finally, our 5\% rule for designating a centre as blinded/not blinded/mixed was arbitrarily selected.

\section{Policy implications}

In breast screening programmes with arbitration of discordant decisions between readers, blinding the second reader to the decision of the first may improve the PPV of breast cancer screening and reduce the number of women recalled for further testing. The results suggest that reader 2 might be influenced by, and conform to, reader 1's decisions when not blinded, particularly if a woman has been recalled by reader 1 (potential alliterative bias). So when reader 2 is not blinded, they appear to be copying some of the recall decisions of reader 1 , and therefore bypassing the arbitration process and increasing recall rates and false positives. A previous study (where the arbitration was in a laboratory rather than screening practice context) predicted similar patterns $[13,14]$. The effect on cancer detection rate is unclear, but the point estimates were higher when blinded in both studies. These results are not generalizable to screening programmes where all discordant decisions are recalled. In that context, previous research has suggested blinding increases cancer detection and false positive recall, whilst maintaining similar PPV.

\section{Conclusions}

Our results suggest that when not blinded reader 2 is influenced by reader 1 's decisions to recall (alliterative bias) which would result in bypassing arbitration and negate some of the benefits of double reading. We found a relationship between blinding the second reader and slightly higher PPV of breast cancer screening, although this analysis may be confounded by other centre characteristics. We would recommend blinded 
over non-blinded double reading in centres that use arbitration of discordant decisions.

Supplementary Information The online version contains supplementary material available at https://doi.org/10.1007/s00330-021-07965-z.

Acknowledgements We are very grateful for the contribution of the staff at all of the centres which participated in the CO-OPS trial. The views expressed in this publication are those of the author(s) and not necessarily those of the NIHR, the UK National Screening Committee, the NHS or the Department of Health and Social Care.

Funding Professor Taylor-Phillips is supported by an NIHR Career Development Fellowship (CDF-2016-09-018). This research was supported by the NIHR Cambridge Biomedical Research Centre (BRC1215-20014).

\section{Declarations}

Guarantor The scientific guarantor of this publication is Professor Sian Taylor-Phillips.

Conflict of interest The authors of this manuscript declare no relationships with any companies whose products or services may be related to the subject matter of the article.

Statistics and biometry David Jenkinson has significant statistical expertise and is a statistician at the University of Warwick and one of the authors. Jennifer Cooper is a Senior Research Associate in Medical Statistics at the University of Bristol. Professor Sian Taylor-Phillips is a Professor of Population Health for Warwick Screening at Warwick Medical School.

Informed consent Written informed consent for the original trial was obtained from each director of breast screening for the CO-OPS Trial (isrctn.org Identifier: ISRCTN46603370). All patient and reader details were de-identified before sending to the researchers.

Ethical approval Institutional Review Board approval for the original trial was obtained from Coventry and Warwickshire National Health Service [NHS] Research Ethics Committee, June 27, 2012, WM/0182.

Study subjects or cohorts overlap None of the outcomes in the current paper have been previously reported. Some study subjects or cohorts have been previously reported in:

Taylor-Phillips S, Wallis MG, Jenkinson D et al (2016) Effect of Using the Same vs Different Order for Second Readings of Screening Mammograms on Rates of Breast Cancer Detection: A Randomized Clinical Trial. JAMA 315:1956-1965

Taylor-Phillips S, Jenkinson D, Stinton C, Wallis MG, Dunn J, Clarke A (2018) Double Reading in Breast Cancer Screening: Cohort Evaluation in the CO-OPS Trial. Radiology 287:749-757

Cooper JA, Jenkinson D, Taylor-Phillips S (2019) Blinding of the second reader in mammography screening: impact on behaviour and cancer detection", Proc. SPIE 10952, Medical Imaging 2019: Image Perception, Observer Performance, and Technology Assessment, 109520T (4 March 2019)

\section{Methodology}

- Observational cohort

- Multicentre study
Open Access This article is licensed under a Creative Commons Attribution 4.0 International License, which permits use, sharing, adaptation, distribution and reproduction in any medium or format, as long as you give appropriate credit to the original author(s) and the source, provide a link to the Creative Commons licence, and indicate if changes were made. The images or other third party material in this article are included in the article's Creative Commons licence, unless indicated otherwise in a credit line to the material. If material is not included in the article's Creative Commons licence and your intended use is not permitted by statutory regulation or exceeds the permitted use, you will need to obtain permission directly from the copyright holder. To view a copy of this licence, visit http://creativecommons.org/licenses/by/4.0/.

\section{References}

1. Perry N, Broeders M, de WolfC, Tornberg S, Holland R, von Karsa L (2008) European guidelines for quality assurance in breast cancer screening and diagnosis. Fourth edition-summary document. Ann Oncol 19:614-622

2. European Commission Initiative on Breast Cancer (2020) European guidelines on breast cancer screening and diagnosis. Available via $\mathrm{https}$ :/healthcare-quality.jrc.ec.europa.eu/european-breast-cancerguidelines. Accessed 16 Oct 2020

3. Thurfjell EL, Lernevall KA, Taube AA (1994) Benefit of independent double reading in a population-based mammography screening program. Radiology 191:241-244

4. Brown J, Bryan S, Warren R (1996) Mammography screening: an incremental cost effectiveness analysis of double versus single reading of mammograms. BMJ 312:809-812

5. Ciatto S, Ambrogetti D, Bonardi R et al (2005) Second reading of screening mammograms increases cancer detection and recall rates. Results in the Florence screening programme. J Med Screen 12: 103-106

6. Coolen AMP, Voogd AC, Strobbe LJ, Louwman MWJ, TjanHeijnen VCG, Duijm LEM (2018) Impact of the second reader on screening outcome at blinded double reading of digital screening mammograms. Br J Cancer 119:503-507

7. Taylor-Phillips S, Jenkinson D, Stinton C, Wallis MG, Dunn J, Clarke A (2018) Double reading in breast cancer screening: cohort evaluation in the CO-OPS Trial. Radiology 287:749-757

8. Leivo T, Salminen T, Sintonen H et al (1999) Incremental costeffectiveness of double-reading mammograms. Breast Cancer Res Treat 54:261-267

9. van Luijt PA, Heijnsdijk EAM, Fracheboud J et al (2016) The distribution of ductal carcinoma in situ (DCIS) grade in 4232 women and its impact on overdiagnosis in breast cancer screening. Breast Cancer Res 18:47-47

10. Taylor-Phillips S, Stinton C (2020) Double reading in breast cancer screening: considerations for policy-making. Br J Radiol 93: 20190610

11. Klompenhouwer EG, Voogd AC, den Heeten GJ et al (2015) Blinded double reading yields a higher programme sensitivity than non-blinded double reading at digital screening mammography: a prospected population based study in the south of The Netherlands. Eur J Cancer 51:391-399

12. Weber RJ, Klompenhouwer EG, Voogd AC, Strobbe LJ, Broeders MJ, Duijm LE (2015) Comparison of the diagnostic workup of women referred at non-blinded or blinded double reading in a population-based screening mammography programme in the south of the Netherlands. Br J Cancer 113:1094-1098

13. Klompenhouwer EG, Voogd AC, den Heeten GJ et al (2015) Discrepant screening mammography assessments at blinded and non-blinded double reading: impact of arbitration by a third reader on screening outcome. Eur Radiol 25:2821-2829 
14. Klompenhouwer EG, Weber RJP, Voogd AC et al (2015) Arbitration of discrepant BI-RADS 0 recalls by a third reader at screening mammography lowers recall rate but not the cancer detection rate and sensitivity at blinded and non-blinded double reading. Breast 24:601-607

15. Smith MJ (1967) Error and variation in diagnostic radiology. Charles C Thomas Publisher, Springfield Illinois

16. Berlin L (2014) Radiologic errors, past, present and future. Diagnosis (Berl) 1:79-84

17. Busby LP, Courtier JL, Glastonbury CM (2018) Bias in radiology: the how and why of misses and misinterpretations. Radiographics 38:236-247

18. Waite S, Scott J, Gale B, Fuchs T, Kolla S, Reede D (2017) Interpretive error in radiology. AJR Am J Roentgenol 208:739-749

19. Bruno MA, Walker EA, Abujudeh HH (2015) Understanding and confronting our mistakes: the epidemiology of error in radiology and strategies for error reduction. Radiographics 35:1668-1676

20. von Elm E, Altman DG, Egger M, Pocock SJ, Gotzsche PC, Vandenbroucke JP (2008) The Strengthening the Reporting of Observational Studies in Epidemiology (STROBE) statement: guidelines for reporting observational studies. J Clin Epidemiol 61:344-349

21. Taylor-Phillips S, Wallis MG, Jenkinson D et al (2016) Effect of using the same vs different order for second readings of screening mammograms on rates of breast cancer detection: a randomized clinical trial. JAMA 315:1956-1965

22. NHS Cancer Screening Programmes (2011) Quality assurance guidelines for breast cancer screening radiology. NHSBSP
Publication No 59. Available via https://assets.publishing.service. gov.uk/government/uploads/system/uploads/attachment_data/file/ 764452/Quality_assurance_guidelines_for_breast_cancer screening_radiology_updated_Dec_2018.pdf. Accessed $1 \overline{8}$ Jan 2019

23. Zhang Z, Parker RMA, Charlton CMJ, Leckie G, Browne WJ (2016) R2MLwiN: a package to run MLwiN from within R. J Stat Softw 72

24. Browne WJ (2017) MCMC estimation in MLwiN v3.00. Centre for Multilevel Modelling, University of Bristol

25. Charlton C, Rasbash J, Browne WJ, Healy M, Cameron B (2017) MLwiN version 3.00. Centre for Multilevel Modelling, University of Bristol

26. Amrhein V, Greenland S, McShane B (2019) Scientists rise up against statistical significance. Nature 567:305-307

27. Wasserstein RL, Schirm AL, Lazar NA (2019) Moving to a world beyond " $p<0.05$ ". Am Stat 73:1-19

28. Colquhoun D (2019) The false positive risk: a proposal concerning what to do about p-values. Am Stat 73:192-201

29. Checka CM, Chun JE, Schnabel FR, Lee J, Toth H (2012) The relationship of mammographic density and age: implications for breast cancer screening. AJR Am J Roentgenol 198:W292-W295

Publisher's note Springer Nature remains neutral with regard to jurisdictional claims in published maps and institutional affiliations. 